\title{
BMJ Open In-Motion-App for remote General Movement Assessment: a multi-site observational study
}

\author{
Lars Adde (1) , , ${ }^{1,2}$ Annemette Brown, ${ }^{3,4}$ Christine van den Broeck, ${ }^{5}$ Kris DeCoen, ${ }^{6}$ \\ Beate Horsberg Eriksen, ${ }^{7}$ Toril Fjørtoft, ${ }^{1,2}$ Daniel Groos, ${ }^{8}$ Espen Alexander F Ihlen, ${ }^{8}$ \\ Siril Osland, ${ }^{2}$ Aurelie Pascal, ${ }^{5}$ Henriette Paulsen, ${ }^{9}$ Ole Morten Skog, ${ }^{10}$ \\ Wiebke Sivertsen, ${ }^{7}$ Ragnhild Støen ${ }^{1,11}$
}

To cite: Adde L, Brown A, van den Broeck C, et al. In-Motion-App for remote General Movement Assessment: a multi-site observational study. BMJ Open 2021;11:e042147. doi:10.1136/ bmjopen-2020-042147

- Prepublication history and supplemental material for this paper is available online. To view these files, please visit the journal online (http://dx.doi. org/10.1136/bmjopen-2020042147).

Received 26 June 2020 Revised 21 January 2021 Accepted 19 February 2021

Check for updates

(C) Author(s) (or their employer(s)) 2021. Re-use permitted under CC BY-NC. No commercial re-use. See rights and permissions. Published by BMJ.

For numbered affiliations see end of article.

Correspondence to

Dr Lars Adde;

lars.adde@ntnu.no

\section{ABSTRACT}

Objectives To determine whether videos taken by parents of their infants' spontaneous movements were in accordance with required standards in the In-Motion-App, and whether the videos could be remotely scored by a trained General Movement Assessment (GMA) observer. Additionally, to assess the feasibility of using home-based video recordings for automated tracking of spontaneous movements, and to examine parents' perceptions and experiences of taking videos in their homes.

Design The study was a multi-centre prospective observational study.

Setting Parents/families of high-risk infants in tertiary care follow-up programmes in Norway, Denmark and Belgium.

Methods Parents/families were asked to video record their baby in accordance with the In-Motion standards which were based on published GMA criteria and criteria covering lighting and stability of smartphone. Videos were evaluated as GMA 'scorable' or 'non-scorable' based on predefined criteria. The accuracy of a 7-point body tracker software was compared with manually annotated body key points. Parents were surveyed about the In-Motion-App information and clarity.

Participants The sample comprised 86 parents/families of high-risk infants.

Results The 86 parent/families returned 130 videos, and $121(96 \%)$ of them were in accordance with the requirements for GMA assessment. The 7-point body tracker software detected more than $80 \%$ of body key point positions correctly. Most families found the instructions for filming their baby easy to follow, and more than $90 \%$ reported that they did not become more worried about their child's development through using the instructions.

Conclusions This study reveals that a short instructional video enabled parents to video record their infant's spontaneous movements in compliance with the standards required for remote GMA. Further, an accurate automated body point software detecting infant body landmarks in smartphone videos will facilitate clinical and research use soon. Home-based video recordings could be performed without worrying parents about their child's development.

Trials registration number NCT03409978.
Strengths and limitations of this study

- A cohort of families of high-risk infants frequently seen in neonatal infant care units follow-up settings.

- In-Motion-App standards for remote General Movement Assessment communicated through a simple and short animated video.

- Data from a motion tracking software on smartphone videos pioneering automatic and markerless infant motion capture.

- Study did not assess sociodemographic factors as reasons for families not to record or return videos.

- Study did not evaluate how markerless infant motion capture on smartphone videos can be used for prediction of cerebral palsy outcome.

\section{INTRODUCTION}

Cerebral Palsy (CP) is the most common physical disability in childhood. Diagnosis is typically set between 12 and 24 months corrected age. ${ }^{1-3}$ Early developmental screening of high-risk infants to predict future neurological impairments is today a priority for clinicians and researchers, and most parents express interest in such neurodevelopmental screening. ${ }^{34}$

The General Movement Assessment (GMA) has been recommended in combination with MRI to achieve a CP diagnosis before 6 months corrected age in infants with newborn-detectable risk factors. ${ }^{3}$ Early detection of CP has the potential to improve the organisation and resources used in follow-up screening at hospitals and reduce medical complications for children with CP. The fidgety type of general movements (GMs) observed at 9-20 weeks' corrected age has shown the highest predictive validity for later CP, compared with the writhing type of GMs observed before 9 weeks corrected age $^{5-8}$ Video recordings for GMA must follow requirements for infant state, position 
and clothing and are scored by certified and trained assessors. ${ }^{9}$ Such trained GMA observers also have the expertise needed to ensure that video recordings fulfil the requirements for a valid GMA. ${ }^{9}$

Access to trained observers using the GMA in hospitalbased follow-up programmes is limited by geographical constraints and lack of GMA expertise. ${ }^{10}$ As healthcare and parents move into the digital age using smartphones to share videos via internet, opportunities to perform remote GMA have developed. Recently, the Baby Moves smartphone app was presented for remote GMA within research settings. ${ }^{10}{ }^{11}$ However, smartphone apps for health data capturing in clinical settings are rarely assessed and usability tested. ${ }^{12}$ To be feasible in a clinical follow-up setting, home-based video recordings must fulfil basic GMA requirements without the need for comprehensive parental training or guiding.

Video recordings by hand-held smartphones introduce movement artefacts in the camera. Computer-based methods for objective detection of infant $\mathrm{GMs}^{13}{ }^{14}$ may be jeopardised by such artefacts. Our research group has recently presented a machine-learning model which predicted CP with high accuracy (sensitivity of $92 \%$, specificity of $81 \%$ ) performed by clinician using a stationary camera, comparable to observational GMA. ${ }^{15}$ Important shortcomings of the method were the need for manual and time-consuming body point annotations, as well as the need for a stationary camera. Hence, an automated 7-point body tracker has been developed by our group and needs to be validated on recordings taken with handheld cameras.

Provided that GMs can be assessed, and computerbased infant body point tracking can be performed on videos taken with a hand-held camera, it is possible to perform remote GMA as well as automated infant body point tracking for a computer-based model for the prediction of CP. Thus, the In-Motion instructional video has been developed so that parents can perform homebased videos with quality standards feasible for remote GMA and automated infant body point tracking. Feasibility of the In-Motion instructional video was assessed in a multi-site study including families of high-risk infants from Norway, Denmark and Belgium.

The main aims of the study are as follows: (1) to determine whether videos taken by parents with a hand-held camera were in accordance with the standards set in the instructional video, and whether the videos could be scored by a trained GMA observer; (2) to assess the accuracy of a 7-point body tracker software based on the same recordings; (3) to describe parents' perceptions of the instructional video and filming their baby in a home environment.

\section{METHODS \\ Design \\ Multi-centre prospective observational study.}

\section{Patient and public involvement}

The study protocol including the parental survey content was developed and designed collaboratively with representatives from The Norwegian Cerebral Palsy organisation and The Norwegian Premature Association.

\section{Participants}

Parents of infants admitted to one of five participating level III-IV neonatal infant care units (NICU) in Norway (3 hospitals including 13, 11 and 4 families, respectively), Denmark (1 hospital including 43 families) and Belgium (1 hospital including 15 families) from 2018 to 2019 (12 months recruitment period) were consecutively recruited at referral to the hospital follow-up programme before discharge from the NICU. Families were recruited based on willingness to participate and the infant being evaluated as at high-risk of CP. In Norway and Denmark inclusion criteria were (1) birth weight $(\mathrm{BW}) \leq 1000 \mathrm{~g}$ (extremely low BW and/or gestational age (GA) $<28$ (extremely low GA), (2) neonatal arterial ischaemic stroke, (3) neonatal encephalopathy, (4) other significant risk factors. In Belgium, only infants with GA $<32$ weeks or with perinatal stroke were included.

\section{Data collection procedure}

Included participants were assisted by a research physiotherapist/paediatrician at the time of inclusion to download and instal the In-Motion-App by smartphone, containing the instructional video from Google Play or iTunes. They could ask any questions about the app and how to manage the software. They got information about the time window for performing two separate video recordings for their infant between $12^{+1}-13^{+6}$ and $14^{+1}-17^{+6}$ weeks post-term age (PTA). ${ }^{11}$ The time points were defined to ensure GM videos from the fidgety movement's period. If no videos were returned from the families before $17^{+6}$ weeks PTA, the local study coordinator contacted the family by phone to ask the reason why they had not uploaded any videos. The app was linked to a secure online server hosted at St. Olavs Hospital in Trondheim, Norway, and was available for i-operating system and Android. After the end of the second time window, the families were contacted by email with a link to an online Norwegian University of Science and Technology (NYNU) survey to collect information about their opinions using the app and the In-Motion instructional video.

\section{In-Motion-App and instructional video}

The In-Motion-App and instructional video was designed by GMA trained personnel (LA, TF, RS, SO) at St. Olavs Hospital in Trondheim, Norway, for parents to give basic insight into recording standards needed for GMA and lighting and stability of camera. It was made as a short animation with simple drawn sequences containing a minimum of text. The instructional video was deployed to the parents by downloading the In-Motion-App developed for this study, and videos could be uploaded to be remotely assessed by a trained GMA observer. 
After downloading the app and getting basic information from the local study coordinator, parents logged into the app with a username and a password. They typed in the first name of their child and the expected date of delivery (due date). The In-Motion-App generated two separate time windows between $12^{+1}-13^{+6}$ and $14^{+1}-17^{+6}$ weeks $\mathrm{PTA}^{11}$ and visualised them in a graphical timeline to show when videos should be taken. A red dot illustrated today's date placed on the timeline, helping parents to plan when to perform the video recordings. In addition, a pop-up message reminded parents to prepare for videos a week before the beginning of each time window. ${ }^{11}$ The In-Motion-App was constructed in such a way that the video recording automatically stopped after $3 \mathrm{~min}$ and asked the parents whether to upload the video or not.

The instructional video was $2 \mathrm{~min}$ and $47 \mathrm{~s} \mathrm{long}$. Before taking videos, parents were told to look through the In-Motion instructional video which was available from the app menu. They could watch the video as many times as they wanted until they felt confident performing the recording. The main themes aimed at ensuring quality standards for remote GMA included: (1) clothing of infant (just a diaper or a onesie), (2) surface/underlay for infant (single-colour blanket or rug), (3) lighting (enough light avoiding sidelight that can cause shadows), (4) state of infant (awake, alert, content, not disturbing baby, no pacifier), (5) positioning (baby on floor-stand next to the baby's feet, whole body must be visible) and (6) length of video ( $3 \mathrm{~min})$. In addition, instructions were provided about how to keep the smartphone steady and ensure that the whole infant body was observable in the video image. Examples of some of the In-Motion instructional video themes are shown in figure 1 . Parents were asked to consecutively upload videos to the server at St. Olavs Hospital in Norway.

\section{Assessment of video quality for remote GMA}

Videos were assessed by a certified GMA observer with respect to the following standards ${ }^{9}:$ (1) GMA standards: active movements (not hypokinetic), supine position, correct state, adequate clothing (diaper or a onesie), no disturbances during recording. (2) Additional In-Motion standards: adequate light, whole body visible, feet of parent visible in video (ensuring correct position of smartphone camera, see picture to the right in figure 1) and camera stability. Based on these standards, a classification was made by the same certified GMA observer as either 'GMA scorable' if all standard criteria were fulfilled or 'GMA non-scorable' if one or more standard criteria were inadequate. In addition, all videos were observed by the same GMA expert who also categorised yes/no whether the hand-held video had optimal stability, events of abrupt displacement, was predominantly unstable, whether an adequate underlay was used (firm, comfortable, large enough) and whether overall video image quality was sufficient (blurred/very blurred).

\section{Assessment of GMs}

All videos classified as 'GMA scorable' were consecutively assessed by one certified and experienced GMA observer that had passed advanced GMs courses under the General Movement Trust (LA). The use of one observer was chosen due to the study design not focusing on GMA and prediction of outcome. The observer had no knowledge about the infant's clinical history. According to Prechtl's method of assessment of GMs, ${ }^{9}$ fidgety movements were classified as continuous $(\mathrm{FM}++)$, intermittent $(\mathrm{FM}+)$, sporadic $(\mathrm{FM}+/-)$, abnormal $(\mathrm{Fa})$ or absent $(\mathrm{FM}-)$.

\section{In-Motion body point tracking}

The infant motion tracker algorithm consists of a convolutional neural network trained on 7-body points on 14900 video frames on high-risk infants that had participated in another study from our group. ${ }^{8}$ For further technical details of the previous trained convolutional neural net, the reader is referred to Groos and Aurlien. ${ }^{16}$ To evaluate the infant motion tracker, 5493 video frames was selected from a subset of 66 videos from 36 infants, recorded by the In-Motion-App by 19 September 2018 . Eighty per cent out of the selected 5493 frames were selected by random. The other $20 \%$ were selected manually in order to include body part occlusions (eg, right wrist occluded behind left wrist) that may be challenging to track. The performance of the infant motion tracker was assessed and reported by the following three steps: first, the automatic motion tracking was performed to detect the position of 7-body points (nose, thorax (centre between shoulders), wrists, pelvis and ankles) in each of the 5493 video frames. Second, all 7-body points in the 5493 selected video frames was manually annotated.
Remove the pacifier

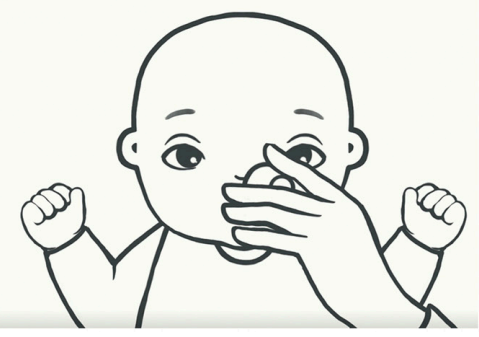

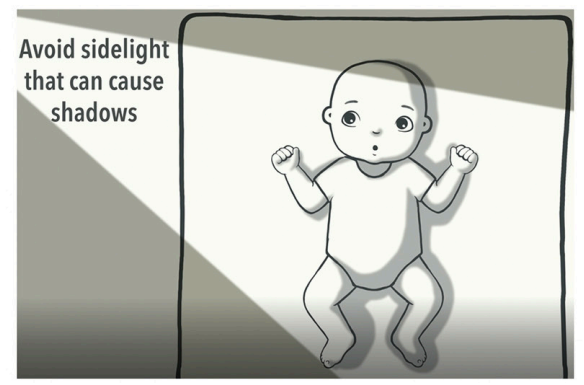

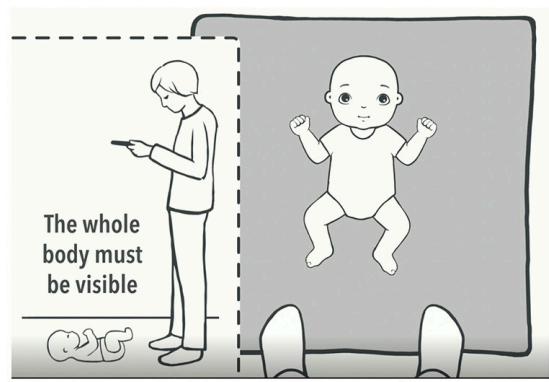

Figure 1 Screenshot of In-Motion instructional video showing examples of information about infant state, lighting and positioning of baby and person filming the baby. 


\begin{tabular}{lc}
\hline & $\%$ \\
\hline Mean & 80.9 \\
Nose & 93.3 \\
Thorax & 93.9 \\
Right wrist & 74.4 \\
Left wrist & 83.1 \\
Pelvis & 63 \\
Right ankle & 79.5 \\
Left ankle & 79.3 \\
\hline
\end{tabular}
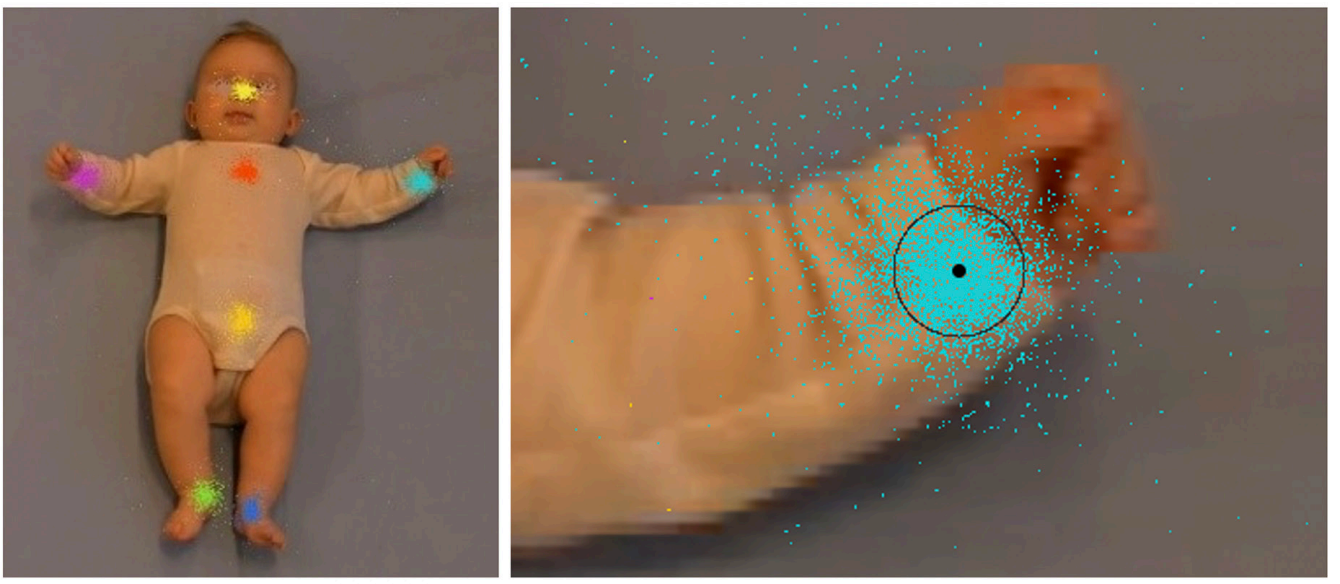

Figure 2 Accuracy of 7 estimated body points compared with manually annotated images. From left: table with proportions of correct detected body point; illustration of the computer-based detections according to 7-body points; the distribution of the left wrist body point detections (blue dots) relative to the manually annotated landmark (black dot) where $10 \%$ of the infant head size is used as a threshold (black circle).

These manually annotations are the ground truth for the evaluation of the infant motion tracker. Third, the performance of the infant motion tracker is reported as percentage of points within a circular area centred at the manually annotated body point for the 5493 frames. In accordance with the established metric for evaluating pose-estimation, ${ }^{17}$ radius of the circular area was set to $10 \%$ of the infant head size and was normalised to adjust for different scaling (ie, video zoom) (figure 2).

\section{Survey}

Parents' opinions about the In-Motion-App and instructional video were collected using SelectSurvey V.4 software (ClassApps, www.classapps.com) in Norway, operated by NTNU. In Denmark, parents' opinions were collected using Smart Trails data management software (MEDEI ApS, www.medei.dk) and in Belgium the survey was collected by post. The survey was sent to the families by a link in an email within 1 week after the last video was returned. The survey questions were customised for the In-Motion-App based on a tool developed by Jin and $\mathrm{Kim}^{18}$ and a survey used in a similar study on the Baby Moves App. ${ }^{11}$ It contained questions based on forced-choice questions covering the themes: (1) In-Motion-App, (2) In-Motion standards for remote GMA and (3) parental worries. A statement was made, and the parents indicated agreement or disagreement with the statement on a 5-point scale (online supplemental appendix 1).

\section{Data analysis}

Data were analysed using SPSS statistics V.26.0 (IBM SPSS Statistics). The data are presented as numbers with proportion (\%) or mean with SD and range. Differences between infants with returned and no returned videos were analysed using the Mann-Whitney $\mathrm{U}$ test for continuous variables and $\chi^{2}$ test and Fisher's exact test for dichotomous variables. The accuracy of the 7-point body tracker was presented as the distribution of body point detections relative to the manually annotated body points, where $10 \%$ of the infant head size was used as a threshold.

\section{RESULTS}

In total, 86 infants/families were recruited and 17 $(19.8 \%)$ out of them did not submit any video (figure 3 ), leaving 69 families with 130 videos for analysis.

Twenty-eight $(32.6 \%)$ families were included from three different hospitals in Norway, $43(50 \%)$ from one hospital in Copenhagen, Denmark and 15 (17.4\%) from one hospital in Gent, Belgium. Infant/family characteristics are shown in table 1.

\section{Video recordings}

Two $(1.5 \%)$ out of 130 videos were shorter than $3 \mathrm{~min}$ ( $1 \mathrm{~min}$ and $12 \mathrm{~s}$ and $2 \mathrm{~min}$ and $17 \mathrm{~s}$ ). The mean PTA at video recording was 14.5 weeks (SD 2.28, range 8.1-23.6 weeks).

Fifteen $(11.7 \%)$ families returned one recording, 49 $(71 \%)$ two recordings, $4(5.8 \%)$ three recordings and $1(1.4 \%)$ six recordings. One-hundred and seventeen $(90 \%)$ videos were returned within the expected time window between $12^{+1}$ and $17^{+6}$ weeks PTA. Two (1.5\%) families returned two and three videos, respectively, which were all taken outside the time window (week 8,10 , and 21 and 23, respectively). Eleven families (8.5\%) with videos from within the requested time window, had additional videos taken outside the expected time windows. Six videos from one family were taken at two different days; four videos in weeks 12 and two videos in week 14 PTA.

\section{Remote GMA}

Among the six videos returned from one family, the first one from each of the 2 days was selected for GMA analysis. Exclusion of the remaining four videos and additional four videos excluded due to PTA outside of the age 


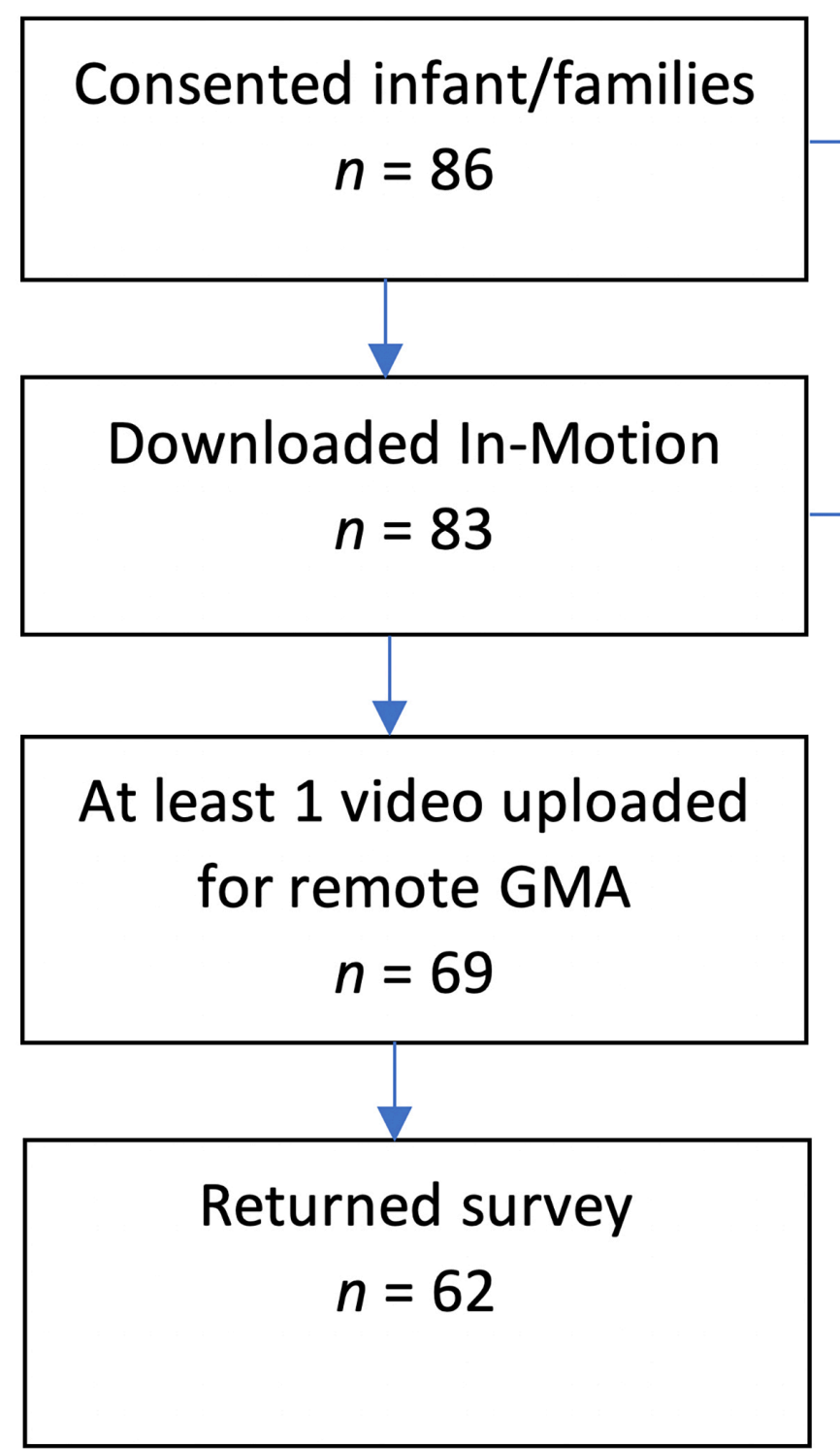

\section{Did not download In-Motion $(n=3)$}

\section{Forgot/busy ( $n=4)$ \\ Social issues $(n=4)$ \\ Unknown reason $(n=6)$}

Figure 3 Flow chart of infant/families with reasons for non-upload of videos. GMA, General Movement Assessment.

required for assessment of FMs gave a total of 122 videos available for quality assessment.

One hundred and twenty-one (99\%) out of 122 videos which were returned within the required time window were classified as GMA scorable. The video that was nonscorable had infant in side lying position. Details about compliance to the In-Motion standards are shown in table 2 .

\section{General Movement Assessment}

Of the 121 videos classified as GMA scorable, $3(2.4 \%)$ videos were classified with exaggerated $(\mathrm{Fa}), 3(2.4 \%)$ with absent (FM-) and $7(5.6 \%)$ with sporadic (FM+/-) FMs. Eighty-seven $(69 \%)$ and $21(16.7 \%)$ videos were classified with intermittent $(\mathrm{FM}+)$ and continuous $(\mathrm{FM}++)$ FMs, respectively.

\section{Computer-based body point tracking}

The proportion of correctly predicted left wrist key point from 5493 tested video images was $83.15 \%$. Details of accuracy of 7-predicted body points and mean value for all points are shown in figure 2 .

\section{Parent responses}

Survey responses were received from 64 (92.8\%) families of the 69 families who returned at least one video. Fiftyfour $(84.3 \%)$ of them observed the instructional video one or two times before filming their baby. No families returned a video without training on filming their baby first.

The majority of the survey respondents found the In-Motion-App easy to use. All respondents agreed or strongly agreed that it was easy to understand how to stand and hold the smartphone during the filming. Details about family responses are shown in table 3. Fiftyseven $(90.5 \%)$ families strongly disagreed, disagreed or neither disagreed nor agreed that they did become more worried about their child's development through using the In-Motion instructions. 
Table 1 Summary of infant/family characteristics

\begin{tabular}{|c|c|c|c|c|}
\hline & $\begin{array}{l}\text { Total N (\%) } \\
(\mathrm{n}=86)\end{array}$ & $\begin{array}{l}N(\%) \text { video } \\
\text { responders ( } n=69)\end{array}$ & $\begin{array}{l}N(\%) \text { no video } \\
\text { responders }(n=17)\end{array}$ & $P$ value \\
\hline \multicolumn{5}{|l|}{ Demographics } \\
\hline Boys, n (\%) & $51(59.3)$ & $42(60.9)$ & $9(52.9)$ & 0.32 \\
\hline Birth weight (BW), mean (SD), g & $1952(1107)$ & $1915(1124)$ & 2105 (1055) & 0.67 \\
\hline Gestational age (GA), mean (SD), weeks & $32.4(5.3)$ & $32.3(5.4)$ & $33.4(4.7)$ & 0.43 \\
\hline \multicolumn{5}{|l|}{ Risk group } \\
\hline $\mathrm{BW} \leq 1000 \mathrm{~g}$ and $/$ or $\mathrm{GA}<28$ & $25(29.1)$ & $20(29)$ & $5(29.4)$ & 0.77 \\
\hline Neonatal arterial ischaemic stroke & $11(12.8)$ & $4(5.8)$ & $7(41.2)$ & 0.001 \\
\hline Hypoxic ischaemic encephalopathy & $20(23.3)$ & $17(24.6)$ & $3(17.6)$ & 0.06 \\
\hline \multirow[t]{2}{*}{ Others } & $32(37.2)$ & $27(39.1)$ & $5(29.4)$ & 0.38 \\
\hline & & \multicolumn{3}{|l|}{ Infant families $(n=63)$} \\
\hline \multicolumn{5}{|l|}{ Sociodemographic data } \\
\hline Mother relation (survey), n (\%) & & $48(76.2)$ & & \\
\hline Married/cohabitant family, n (\%) & & $59(93.7)$ & & \\
\hline Age mother/farther, mean (SD, range) & & $31.8(5.5,21-6)$ & & \\
\hline Age farther, mean (SD, range) & & $33.9(6.9,22-59)$ & & \\
\hline Single child, n (\%) & & $32(51.6)$ & & \\
\hline \multicolumn{5}{|l|}{ iOS vs Android } \\
\hline iOS users, n (\%) & & 41 (65.1) & & \\
\hline
\end{tabular}

iOS, i-operating system.

\section{DISCUSSION}

In this study, more than $95 \%$ of families with high-risk infants filming their baby at home, returned at least one video that was in accordance with the In-Motion standards for remote GMA. Most families found the In-Motion-App easy to use and the instructions for filming easy to follow, and less than $10 \%$ of respondents became worried through using the In-Motion-App. Despite the use of hand-held smartphones introducing movement artefacts in the video image, our computer-based 7-point body tracker detected positions of the body points with high accuracy. To the best of our knowledge, this is the first automatic infant body point tracker that is tested on video recordings from hand-held smartphones.
This study has several strengths. First, it included families of high-risk infants frequently seen in NICU follow-up settings. We argue that this makes our findings robust and generalisable to comparable clinical settings. Second, the communication of In-Motion standards through a simple and short animated video, makes the instructions easily applicable to a broad range of different clinical settings. Furthermore, the accuracy of the 7-point body tracker software with the use of smartphone videos makes it pioneering in the field of automatic and markerless infant motion capture compared with other studies. ${ }^{19} 20$ This facilitates further development of methods for early automated detection of CP based on smartphone videos. Finally, the design of the study using an experienced and

\begin{tabular}{|c|c|c|c|c|c|c|c|c|}
\hline & $\begin{array}{l}\text { Active } \\
\text { movements (not } \\
\text { hypokinetic) }\end{array}$ & $\begin{array}{l}\text { Supine } \\
\text { position }\end{array}$ & Correct state & $\begin{array}{l}\text { No disturbances } \\
\text { during recording }\end{array}$ & $\begin{array}{l}\text { Adequate } \\
\text { clothing }\end{array}$ & $\begin{array}{l}\text { Adequate } \\
\text { light }\end{array}$ & $\begin{array}{l}\text { Whole } \\
\text { body } \\
\text { visible }\end{array}$ & $\begin{array}{l}\text { Feet of } \\
\text { parents } \\
\text { visible }\end{array}$ \\
\hline \multirow[t]{3}{*}{ N (\%) } & $126(100)$ & 125 (99.2) & $122(96.8)$ & $124(98.4)$ & 125 (99.2) & $124(98.4)$ & $124(98.4)$ & $116(92.1)^{\star}$ \\
\hline & & $\begin{array}{l}\text { Optimal } \\
\text { stability }\end{array}$ & $\begin{array}{l}\text { Abrupt } \\
\text { displacement }\end{array}$ & $\begin{array}{l}\text { Predominantly } \\
\text { unstable }\end{array}$ & $\begin{array}{l}\text { Correct } \\
\text { base of } \\
\text { support }\end{array}$ & & $\begin{array}{l}\text { Image } \\
\text { quality }\end{array}$ & \\
\hline & & & & & & Clear & Blurred & Very blurred \\
\hline $\mathrm{N}(\%)$ & & $80(63.5)$ & $26(20.6)$ & $22(17.5)$ & 119 (94.4) & $114(90.5)$ & $11(8.7)$ & $1(0.8)$ \\
\hline
\end{tabular}

*Three missing data. 


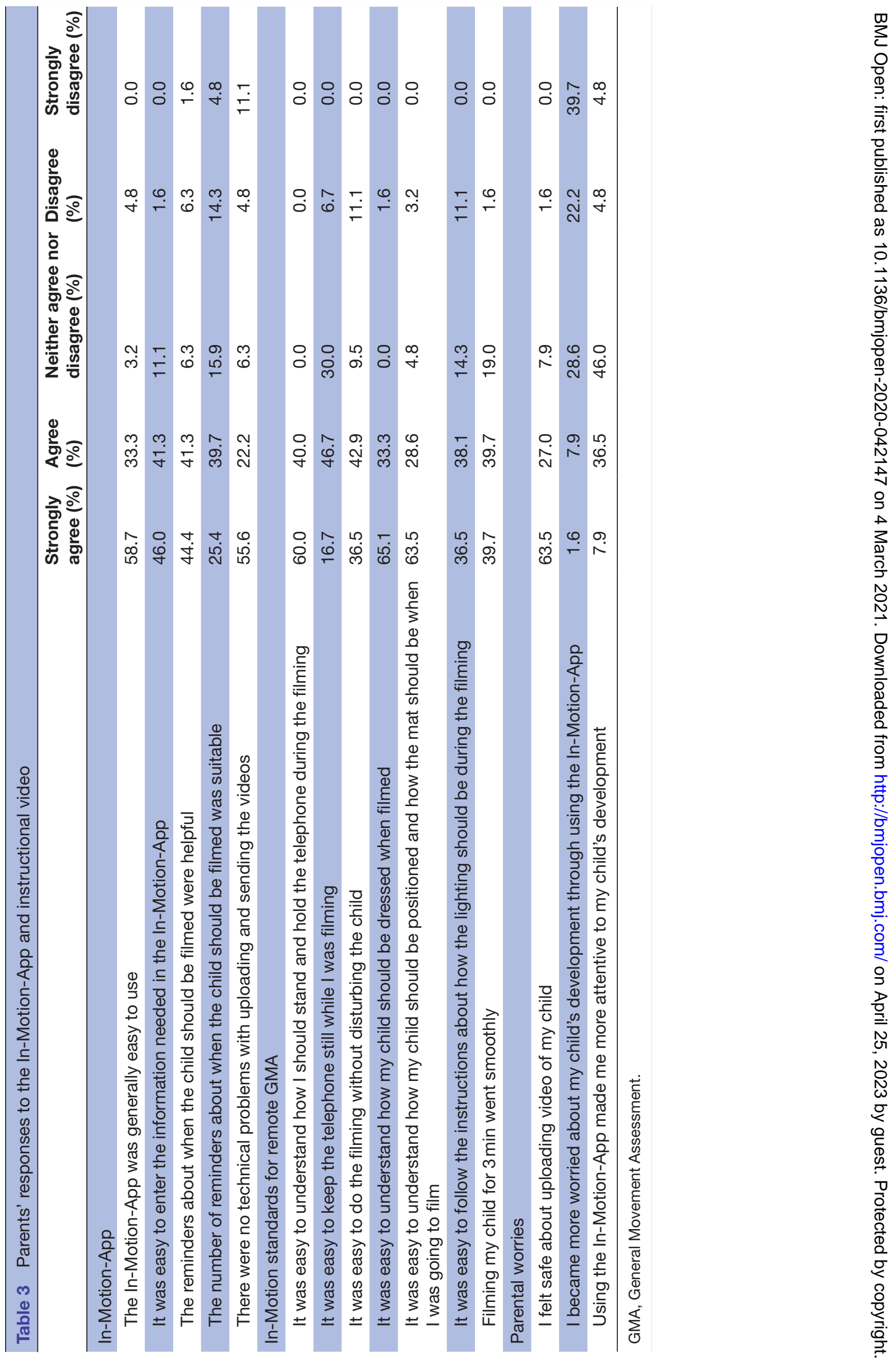


certified GMA observer for evaluation of video quality and a survey with very high response rate, makes the study quality high and the results trustworthy.

There are also several limitations. First, almost $20 \%$ of the included families did not return any video. This study was not designed to evaluate reasons for not recording or returning videos. We can, therefore, only conclude on the quality of returned videos. The questions in our survey may also have limitations, mainly covering topics favourable to participants returning videos, participating in follow-up and smartphone usage, giving little or reduced information about responders with low mobile health technology usage. Hence, problems encountered by families who did not record or return any video need to be further explored. Our findings are in accordance with the study by Kwong et al, ${ }^{11}$ where $24 \%$ of families did not return any video using the Baby Moves App. These findings indicate that home-based video recordings for remote GMA is not a solution for all families and that it might be difficult in a clinical setting to know beforehand which families will return a video or not. Furthermore, 13 (19\%) families returned one or several videos outside the required time window needed for a valid GMA. More than $90 \%$ of respondents found the reminders in the app helpful, but one-fifth disagreed that the number of reminders were appropriate. These findings indicate a limitation in the design of the app reminders and lack of programmed filming windows parameters. These app functionalities need to be improved in a process involving the users.

Second, our study comprised five different hospital sites in three different countries, and information provided to families when downloading the app may have differed. Additionally, there is a risk that the research personnel could have given more information and assistance to families than will be common in an ordinary clinical setting. Our study setting could therefore be in slight contrast to an ordinary clinical setting, where adapted and flexible family information is needed due to challenges in reduced participation in neurodevelopmental follow-up ${ }^{21}$ and racial and socioeconomic differences in mobile health technology usage. ${ }^{22}$

Third, even though almost $20 \%$ of the hand-held videos in our study were classified as predominantly unstable, the GMA expert considered the videos to be GMA scorable. There is a risk that more videos might have been classified as non-scorable by another GMA observer with less training and experience or if there had been several GMA experts observing the same videos. To the best of our knowledge, there is only one study protocol planning to assess the predictive validity of GMA from videos taken with hand-held smartphones for CP outcome. ${ }^{10}$ Hence, further studies on the use of smartphones for GMA are needed.

Finally, our computer-based 7-point body tracker showed accurate estimations compared with manual annotations on the video image. However, further studies must explore how selection of body points, tracked body point accuracy and movement artefacts in camera will influence a machine-learning model for prediction of $\mathrm{CP}$ from smartphone video recordings.

This study facilitates and contributes to the use of smartphone technology for video recordings and remote GMA. Consequently, it will contribute to giving high-risk infants and their families equal access to GMA as an accurate method for early identification of CP, without geographical constraints. The use of early remote medical assessment will improve the organisation and resources used in follow-up screening at hospitals and have the potential to reduce medical complications for children with $\mathrm{CP}$ due to early detection. A clinical feasible computer-based movement analysis with equal accuracy as GMA, will greatly reduce the need for specialised GMA observers and provide an innovative resource-effective diagnostic measure.

\section{Author affiliations}

${ }^{1}$ Department of Clinical and Molecular Medicine, Norwegian University of Science and Technology, Trondheim, Norway

${ }^{2}$ Clinic of Clinical Services, St. Olavs Hospital, Trondheim University Hospital, Trondheim, Norway

${ }^{3}$ Department of Health Sciences, Lund University, Lund, Sweden

${ }^{4}$ Department of Pediatric and Adolescent and Department of Neurology and Physiotherapy, Copenhagen University Hospital, Nordsjællands Hospital, Hillerød, Denmark

${ }^{5}$ Department of Rehabilitation Sciences, Ghent University, Ghent, Belgium ${ }^{6}$ Department of Neonatology, University Hospital Ghent, Gent, Belgium ${ }^{7}$ Department of Pediatrics, Møre og Romsdal Hospital Trust, Ålesund Hospital, Ålesund, Norway

${ }^{8}$ Department of Neuromedicine and Movement Science, Norwegian University of Science and Technology, Trondheim, Norway

${ }^{9}$ Department of Physiotherapy and Rehabilitation, Vestfold Hospital Trust, Tønsberg, Norway

${ }^{10}$ Habilitation Center, Vestfold Hospital Trust, Tønsberg, Norway

${ }^{11}$ Department of Neonatology, St. Olavs Hospital, Trondheim University Hospital, Trondheim, Norway

Acknowledgements We would like to thank all the families that participated in this study. We acknowledge the support from St. Olavs Hospital, The Liaison Committee between the Central Norway Regional Health Authority and the Norwegian Open Al-Lab, Norwegian University of Science and Technology, Trondheim, Norway. Thanks also to Mia Ortved Bjerager consultant neonatologist and the neonatal ward staff at Nordsjællands Hospital in Denmark for their valuable contribution to this study.

Contributors Conceptualisation: LA, RS, HP, OMS, TF, SO, WS, BHE, CvdB. Data curation: LA, RS, EAFI, DG. Formal analysis: LA, RS, EAFI, DG. Funding acquisition: LA, RS. Methodology: LA, RS, EAFI, DG, TF, SO. Project administration: LA, BHE, HP, AP, AB, CvdB, RS, KD. Resources: LA, TF, SO, AB, AP, HP, OMS, BHE, WS, KD. Writingoriginal draft: LA, RS, EAFI, DG. Writing-review and editing: LA, RS, EAFI, DG, TF, S0, BHE, WS, AB, AP, CvdB, HP, OMS, KD.

Funding This work was supported by grants from St. Olavs Hospital, Trondheim, Norway and The Liaison Committee between the Central Norway Regional Health Authority and the Norwegian University of Science and Technology, Trondheim, Norway (S0: 90056100). In Denmark the study was supported by grants from Nordsjællands Hospital, University Hospital of Copenhagen, Hillerød (PSP E-20231-18-01).

\section{Competing interests None declared.}

Patient consent for publication Obtained in all participants.

Ethics approval All infant/families provided written informed consent and ethics was approved by the regional committee for medical and health research ethics (REC Central-Committee 2017/913) in Norway. The study sites in Denmark and Belgium also had approvals from their local Institutional Review boards. 
Provenance and peer review Not commissioned; externally peer reviewed.

Data availability statement No data are available. Data from this study are not available for sharing due to ethical approval requirements. Researchers interested in collaboration should contact the corresponding author with their expression of interest.

Supplemental material This content has been supplied by the author(s). It has not been vetted by BMJ Publishing Group Limited (BMJ) and may not have been peer-reviewed. Any opinions or recommendations discussed are solely those of the author(s) and are not endorsed by BMJ. BMJ disclaims all liability and responsibility arising from any reliance placed on the content. Where the content includes any translated material, BMJ does not warrant the accuracy and reliability of the translations (including but not limited to local regulations, clinical guidelines, terminology, drug names and drug dosages), and is not responsible for any error and/or omissions arising from translation and adaptation or otherwise.

Open access This is an open access article distributed in accordance with the Creative Commons Attribution Non Commercial (CC BY-NC 4.0) license, which permits others to distribute, remix, adapt, build upon this work non-commercially, and license their derivative works on different terms, provided the original work is properly cited, appropriate credit is given, any changes made indicated, and the use is non-commercial. See: http://creativecommons.org/licenses/by-nc/4.0/.

\section{ORCID iD}

Lars Adde http://orcid.org/0000-0001-5532-0034

\section{REFERENCES}

1 Granild-Jensen JB, Rackauskaite G, Flachs EM, et al. Predictors for early diagnosis of cerebral palsy from national registry data. Dev Med Child Neurol 2015;57:931-5.

2 Hubermann L, Boychuck Z, Shevell M, et al. Age at referral of children for initial diagnosis of cerebral palsy and rehabilitation: current practices. J Child Neurol 2016;31:364-9.

3 Novak I, Morgan C, Adde L, et al. Early, accurate diagnosis and early intervention in cerebral palsy: advances in diagnosis and treatment. JAMA Pediatr 2017;171:897-907.

4 Dorner RA, Boss RD, Burton VJ, et al. Parent preferences for neurodevelopmental screening in the neonatal intensive care unit. Dev Med Child Neurol 2020;62:500-5.

5 Kwong AKL, Fitzgerald TL, Doyle LW, et al. Predictive validity of spontaneous early infant movement for later cerebral palsy: a systematic review. Dev Med Child Neurol 2018;60:480-9.

6 Bosanquet M, Copeland L, Ware R, et al. A systematic review of tests to predict cerebral palsy in young children. Dev Med Child Neurol 2013;55:418-26.

7 Darsaklis V, Snider LM, Majnemer A, et al. Predictive validity of Prechtl's method on the qualitative assessment of general movements: a systematic review of the evidence. Dev Med Child Neurol 2011;53:896-906.

8 Støen R, Boswell L, de Regnier R-A, et al. The predictive accuracy of the general movement assessment for cerebral palsy: a prospective, observational study of high-risk infants in a clinical follow-up setting. $J$ Clin Med 2019;8. doi:10.3390/jcm8111790. [Epub ahead of print: $25102019]$

9 Einspieler C, Prechtl HF, Bos A, et al. Prechtl's method on the qualitative assessment of general movements in preterm, term and young infants. London: Mac Keith Press, 2004.

10 Spittle AJ, Olsen J, Kwong A, et al. The Baby Moves prospective cohort study protocol: using a smartphone application with the General Movements Assessment to predict neurodevelopmental outcomes at age 2 years for extremely preterm or extremely low birthweight infants. BMJ Open 2016;6:e013446.

11 Kwong AK, Eeles AL, Olsen JE, et al. The baby moves smartphone APP for general movements assessment: engagement amongst extremely preterm and term-born infants in a state-wide geographical study. J Paediatr Child Health 2019;55:548-54.

12 McCartney M. How do we know whether medical apps work? BMJ 2013;346:f1811.

13 Marcroft C, Khan A, Embleton ND, et al. Movement recognition technology as a method of assessing spontaneous General movements in high risk infants. Front Neurol 2014;5:1-9.

14 Cabon S, Porée F, Simon A, et al. Video and audio processing in paediatrics: a review. Physiol Meas 2019;40:02TR.

15 Ihlen EAF, Støen R, Boswell L, et al. Machine learning of infant spontaneous movements for the early prediction of cerebral palsy: a multi-site cohort study. J Clin Med 2019;9. doi:10.3390/jcm9010005. [Epub ahead of print: 1812 2019].

16 Groos D, Aurlien K. Infant body part tracking in Videos using deep Learning-Facilitating early detection of cerebral palsy: NTNU 2018.

17 Groos D, Ramampiaro H, Ihlen EAF. EfficientPose: scalable singleperson pose estimation. Applied Intelligence 2020;190.

$18 \mathrm{Jin}$ M, Kim J. Development and evaluation of an evaluation tool for healthcare smartphone applications. Telemed J E Health 2015;21:831-7.

19 Sciortino G, Farinella GM, Battiato S, et al, eds. On the estimation of children's poses. International conference on image analysis and processing. New York: Springer, 2017.

20 Hesse N, Bodensteiner C, Arens M, eds. Computer vision for medical infant motion analysis: state of the art and rgb-d data set. Proceedings of the European conference on computer vision (ECCV). Munich, Germany: Springer, 2018.

21 L Orton J, McGinley JL, Fox LM, et al. Challenges of neurodevelopmental follow-up for extremely preterm infants at two years. Early Hum Dev 2015;91:689-94.

22 Hamilton EC, Saiyed F, Miller CC, et al. The digital divide in adoption and use of mobile health technology among caregivers of pediatric surgery patients. J Pediatr Surg 2018;53:1478-93. 